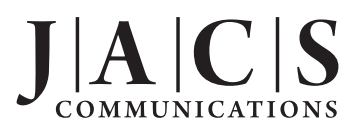

Published on Web 09/23/2009

\title{
Size, Shape, and Internal Atomic Ordering of Nanocrystals by Atomic Pair Distribution Functions: A Comparative Study of $\gamma-\mathrm{Fe}_{2} \mathrm{O}_{3}$ Nanosized Spheres and Tetrapods
}

\author{
Valeri Petkov, ${ }^{*, \dagger}$ P. Davide Cozzoli, ${ }^{\ddagger} \S$ Raffaella Buonsanti, ${ }^{\ddagger, \S}$ Roberto Cingolani, ${ }^{\S, l}$ and Yang Ren ${ }^{\perp}$ \\ Department of Physics, Central Michigan University, Mt. Pleasant, Michigan 48858, Scuola Superiore ISUFI - \\ University of Salento, via per Arnesano km 5, 73100 Lecce, Italy, National Nanotechnology Laboratory of \\ CNR-INFM, Unità di Ricerca IIT, via per Arnesano Km 5, 73100 Lecce, Italy, Fondazione Istituto Italiano di \\ Tecnologia, IIT, Via Morego 30 - 16163 Genova, Italy, and Advanced Photon Source, Argonne National \\ Laboratory, Argonne Illinois 60439
}

Received August 10, 2009; E-mail: petkov@ phy.cmich.edu

A good knowledge of the size, shape, and internal atomic ordering (i.e., atomic positions) of polycrystals is a crucial prerequisite to understanding, predicting, and practically exploiting many of their chemical and physical properties. For micrometerscale polycrystals, this structural information has traditionally been obtained from the analysis of the positions, intensities, and shapes of the Bragg peaks in their powder X-ray diffraction (XRD) patterns. ${ }^{1}$ Recently, a wealth of synthetic success has led to the availability of a broad variety of elaborate nanometer-scale crystals, the in-depth compositional-properties elucidation of which requires

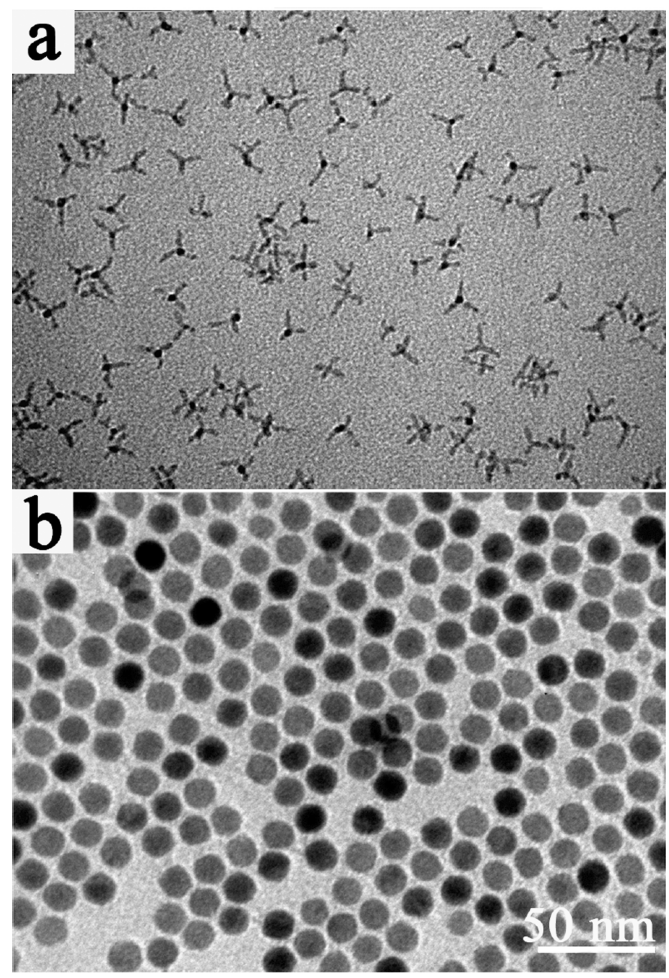

Figure 1. TEM images of $\gamma-\mathrm{Fe}_{2} \mathrm{O}_{3}$ NCs: TPs (a) and SPs (b).

precise structural knowledge. However, in the case of nanocrystal (NCs) effects arising from the inherently very small crystallite sizes, the large surface-to-volume ratio, near-surface relaxation, and local

Central Michigan University.

Scuola Superiore ISUFI - University of Salento.

\& Unità di Ricerca IIT.

"Istituto Italiano di Tecnologia, IIT

${ }^{\perp}$ Argonne National Laboratory. lattice distortions smear out the Bragg reflections in the XRD patterns, ultimately rendering their interpretation very difficult, or even impossible. The problem has been addressed by applying the so-called Debye Function Analysis (DFA). ${ }^{2}$ It involves a simulation of a part of the raw XRD pattern of NCs using distributions of interatomic distances from finite-size structure models of NCs. Recently, another nontraditional approach involving high-energy powder XRD coupled to atomic pair distribution function (PDF) analysis has shown to be particularly well suited for determining the internal atomic ordering of NCs. ${ }^{3}$ The great performance of the approach stems from the fact that it takes into account both the Bragg-like and the diffuse components of the XRD data over an extended range of scattering vectors and not just a part of that range. The extended XRD data are then normalized to electron units,

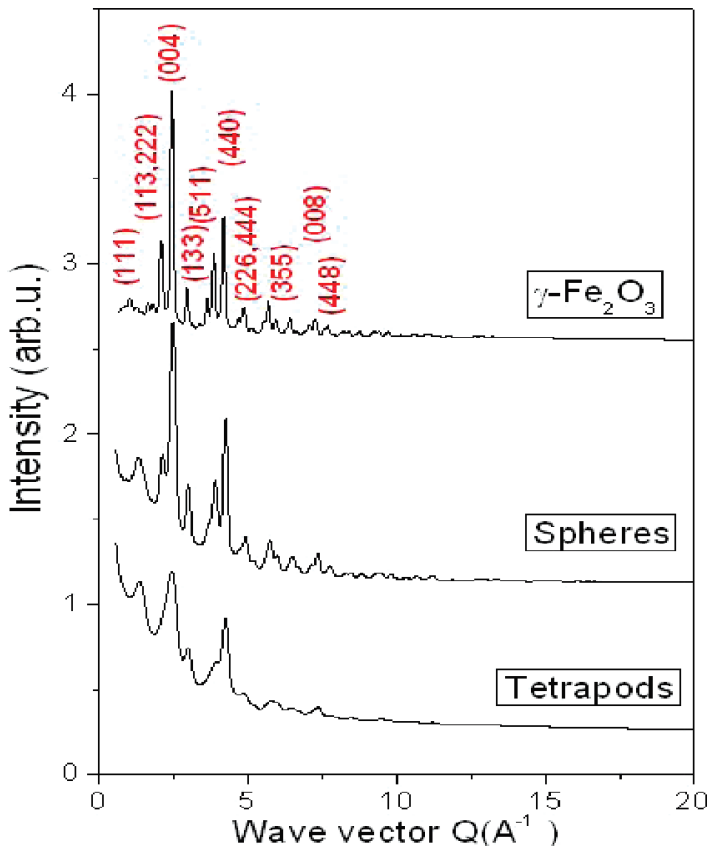

Figure 2. Synchrotron XRD patterns for micrometer-sized polycrystalline $\gamma-\mathrm{Fe}_{2} \mathrm{O}_{3}$ standard (top), spherically (middle), and tetrapod-shaped (bottom) $\gamma-\mathrm{Fe}_{2} \mathrm{O}_{3}$ NCs. Bragg peaks are indexed in the cubic-type (S.G. $F d \overline{3} m$ ) structure of $\gamma-\mathrm{Fe}_{2} \mathrm{O}_{3}$ spinel.

subjected to a Fourier transformation, and analyzed in real space. ${ }^{3}$ In this way, ambiguities arising from experimental artifacts, such as background scattering, ${ }^{2}$ are eliminated. Also, model distributions of interatomic distances can be compared directly to well resolved experimental distributions of such distances and not to a smeared 


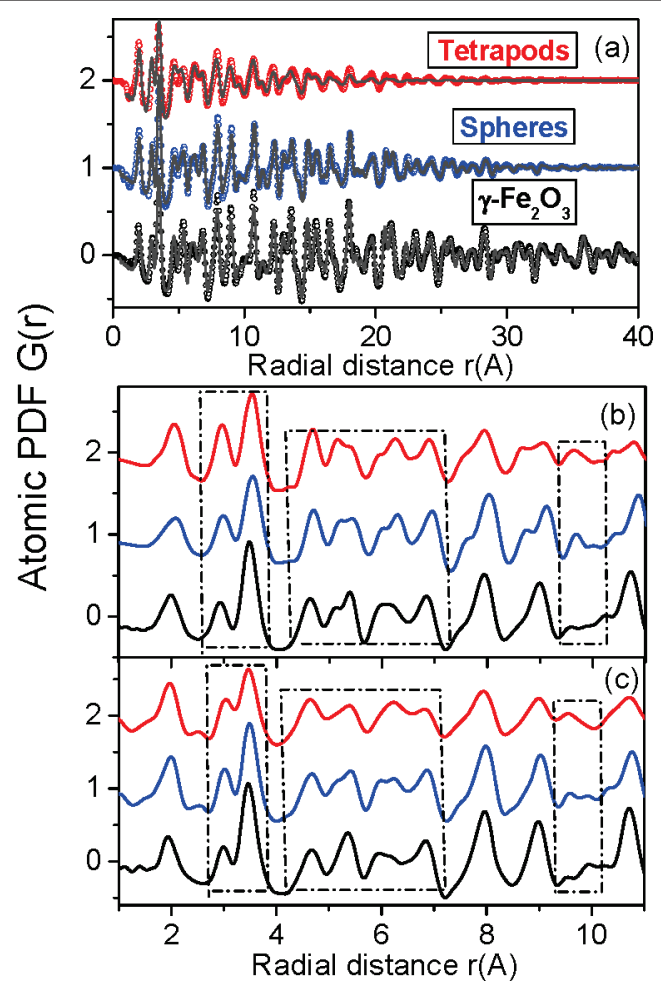

Figure 3. (a) Experimental atomic PDFs for polycrystalline $\gamma-\mathrm{Fe}_{2} \mathrm{O}_{3}$ (black symbols), spherically (blue symbols), and tetrapod-shaped (red symbols) $\gamma-\mathrm{Fe}_{2} \mathrm{O}_{3}$ NCs. Best fits (solid lines) based on a continuous spinel-type (S.G. $F d \overline{3} m$ ) lattice model, as explained in the text, are also shown. (b) Low-r part of model atomic PDFs for polycrystalline $\gamma-\mathrm{Fe}_{2} \mathrm{O}_{3}$ (black line), SPs (blue line), and TPs (red line). The model PDFs are computed from the finite-size atomic configurations shown in Figure 4. (c) Low-r part of the experimental PDFs. The PDF peaks whose relative intensities are correlated with the NC shape are outlined with broken lines.

derivative of them, unlike what is done in DFA. The potential of the PDF approach to reveal the size and, especially, shape of NCs, however, has not been so far proven to be viable and, therefore, remains largely unutilized.

Here, we show that the atomic PDF approach is indeed sensitive to the geometry of $\mathrm{NCs}$ and, therefore, can be used to not only determine their internal atomic ordering but also assess their size and shape. As a proof-of-concept here we report results from a PDF study of cubic, inverse spinel $\gamma-\mathrm{Fe}_{2} \mathrm{O}_{3}$ (maghemite) NCs shaped as spheres (SPs) and tetrapods (TPs). The samples were synthesized by reacting $\mathrm{Fe}(\mathrm{CO})_{5}$ at $240-300{ }^{\circ} \mathrm{C}$ in octadecenediluted mixtures of oleic acid, oleylamine, and hexadecan-1,2-diol as reactivity moderators and shape-controlling agents ${ }^{4}$ (see Supporting Information (SI)). These NCs are important representatives of the technologically valuable class of nanosized transition-metal ferrites that are widely studied for catalysis, high-density recording media, and biomedical applications. ${ }^{5}$ In addition, for the purposes of the present study, cubic-phase NCs were selected because of their highly symmetric internal atomic ordering, i.e., because they lack any inherent structural anisotropy. This allowed the impact of the NC shape anisotropy alone on the PDF data to be studied and clearly demonstrated.

Figure 1 presents low-magnification transmission electron microscopy (TEM) images of $\gamma-\mathrm{Fe}_{2} \mathrm{O}_{3} \mathrm{NCs}$ studied here. TPs (Figure 1a) appear as branched objects made of four arms with a length and diameter of $10.7 \pm 1.2$ and $3.2 \pm 0.5 \mathrm{~nm}$, respectively. The arms depart at tetrahedral angles $\left(\sim 109^{\circ}\right)$ out of a common branching point, as verified by electron holography studies. ${ }^{4}$ The fourth TP arm points upward almost parallel to the electron beam and produces a darker TEM contrast at the projected TP center. Phase-contrast high-resolution TEM studies indicated that the individual TPs are single-crystalline, and their arms are oriented along four out of the eight equivalent [111]-type directions of the cubic spinel lattice. ${ }^{4}$ SPs (Figure $1 \mathrm{~b}$ ) are rather monodisperse with an average diameter of $13.6 \pm 1.2 \mathrm{~nm}$ (see SI).

Figure 2 shows the experimental powder XRD patterns for $\gamma-\mathrm{Fe}_{2} \mathrm{O}_{3}$ SPs, TPs, and a polycrystalline $\gamma-\mathrm{Fe}_{2} \mathrm{O}_{3}$ standard. Thanks to the use of high-energy synchrotron X-rays (see SI) the XRD patterns are measured over an extended region of scattering vectors, $Q$. This is essential for the success of PDF data analysis. ${ }^{3}$ The XRD pattern for the standard (Figure 2, top trace) shows several sharp Bragg peaks reflecting the isotropic shape and $\mu \mathrm{m}$ size of its unstrained crystallites. The peaks can be indexed in the cubic lattice (S.G. $F d \overline{3} m$ ) of the well-known spinel-type structure of $\gamma-\mathrm{Fe}_{2} \mathrm{O}_{3}$. In this structure $\mathrm{Fe}$ atoms occupy sites with tetrahedral and octahedral oxygen coordination in a ratio of 1 to 2 , respectively, with some of the sites being vacant. ${ }^{6 a}$ When the vacancies are ordered, the symmetry of the spinel structure reduces to $P 4_{1} 22_{1} 2$. $^{6 \mathrm{~b}}$ The XRD patterns for $\gamma-\mathrm{Fe}_{2} \mathrm{O}_{3} \mathrm{NCs}$ (Figure 2, middle and bottom traces) are rather diffuse and show only a few broad Bragg-like peaks that overlap considerably for the SPs and are almost unresolved in the case of TPs. The diffuse features of XRD patterns for SPs and TPs were previously considered as a signature of NC shape anisotropy but, however, could not be interpreted unambiguously even with the help of atomistic simulations. ${ }^{4}$ The corresponding atomic PDFs shown in Figure 3, however, exhibit a series of sharp peaks which, as demonstrated below, can be conveniently exploited for both assessing the geometry and determining the internal atomic ordering of NCs.

First, the atomic PDF for polycrystalline $\gamma-\mathrm{Fe}_{2} \mathrm{O}_{3}$ shows a series of sharp peaks, each reflecting a particular atomic coordination sphere, to high interatomic distances, as it should be with a material possessing a long-range ( $\sim \mu \mathrm{m}$ scale) atomic order. The PDFs for $\gamma-\mathrm{Fe}_{2} \mathrm{O}_{3} \mathrm{NCs}$ also show several sharp peaks. Those peaks, however, decay to zero much faster (see Figure 3a) reflecting the limited length ( $\sim$ nm scale) of structural coherence (LSC) in the NCs. A precise estimate of that length in NC SPs and TPs, including taking into account the XRD instrumental resolution, can be done following the procedure of Ergun et al. ${ }^{7}$ The procedure assumes that the LSC in NCs causes an exponential decay of the PDF data of the type $\exp (-\alpha r)$, where $r$ is the radial distance and $2 / \alpha$ is the average LSC. Other somewhat more sophisticated but similar procedures have also been suggested. ${ }^{8}$ The application of Ergun's procedure, as described in Gateshki et al., ${ }^{9}$ shows that the LSC, also known as the size of crystallite domains that scatter X-rays coherently, is $\sim 3 \mathrm{~nm}$ for the TPs and $\sim 4 \mathrm{~nm}$ for the SPs. Note the diameter of TP arms is $\sim 3 \mathrm{~nm}$, suggesting that the arm diameter imposes a limit to the "crystallite domain size" in TPs. As it can be expected, the "crystallite domain size" is larger $(\sim 4 \mathrm{~nm})$ in the larger SPs, yet it is smaller than their TEM-estimated size $(\sim 13.6$ $\mathrm{nm}$ ). The presence of lattice stain is most likely what limits the LSC in the SPs. ${ }^{2}$ Clearly, atomic PDFs, in particular the way they decay to zero, reflect the LSC in NCs and, hence, can be used to estimate it. Note, just like in polycrystals, the LSC in NCs can be set by the (nano)crystallite size and/or lattice defects/strain.

Second, thanks to the special properties of Fourier transformation, the rather smeared XRD patterns of NCs appear as well resolved distributions of interatomic distances with distinctive profiles. This allows an unambiguous identification of NC shape. In particular, the profiles of the PDFs for SPs and TPs differ in the relative intensities of several corresponding peaks. The differences are exactly of the type predicted by structure models of real-size SP 
(a)

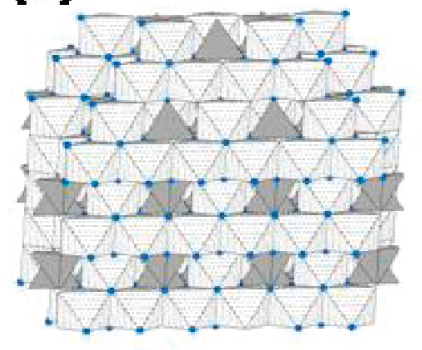

(b)

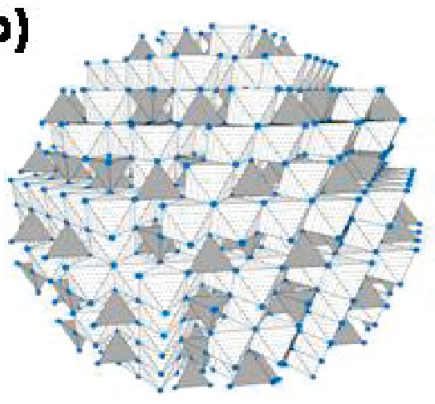

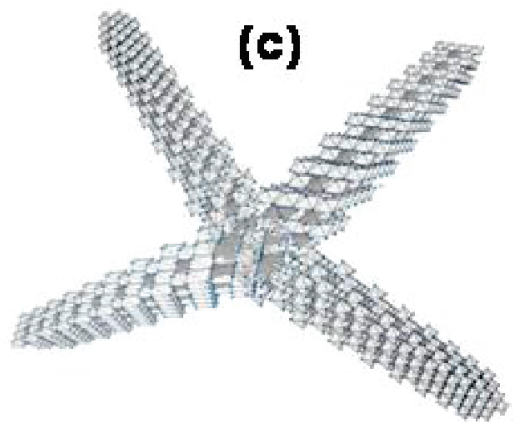

Figure 4. Fragment from the continuous spinel lattice of polycrystalline $\gamma-\mathrm{Fe}_{2} \mathrm{O}_{3}$ standard (a), nanosized $\gamma-\mathrm{Fe}_{2} \mathrm{O}_{3} \mathrm{SP}$ (b), and TP (c). Fe- $\mathrm{O}_{6}$ octahedra are in light gray, $\mathrm{Fe}-\mathrm{O}_{4}$ tetrahedra are in dark gray, while $\mathrm{O}$ atoms are shown as blue dots. The TP arms are oriented along the [111]-type of the spinel lattice (S.G. Fd $\overline{3} m$ ) as suggested by high-resolution TEM. ${ }^{4}$

and $\mathrm{TP} \gamma-\mathrm{Fe}_{2} \mathrm{O}_{3} \mathrm{NCs}$ shown in Figure $4 \mathrm{~b}$ and $4 \mathrm{c}$, respectively. The models are built of Fe-based octahedral and tetrahedral units arranged according the symmetry of the cubic (S.G. $F d \overline{3} m$ ) spineltype lattice. Hence, the surface of model SPs and TPs is terminated with $\mathrm{O}$ atoms from these structural units, just like that of the real SPs and TPs studied here. ${ }^{10}$ Since the polycrystalline $\gamma-\mathrm{Fe}_{2} \mathrm{O}_{3}$, nanosized SPs and TPs are characterized by markedly different surface to volume ratios, the relative $\mathrm{O}$ to $\mathrm{Fe}$ contents in the three materials are very different. As a result, the number of $\mathrm{O}$ involving correlations $(\mathrm{Fe}-\mathrm{O}$ and $\mathrm{O}-\mathrm{O}$ ) that contribute to the different coordination spheres (e.g., PDF peaks) of the three samples appears different, although they all share the same type of internal atomic ordering (see below). As expected, the more anisotropic the NC shape (cf. TP vs SP), the stronger the deviation of the profile of the respective PDF from that of the polycrystalline standard. If NCs are made of a material with intrinsic structural anisotropy (i.e., low crystallographic symmetry), the differences in the PDF profiles may be enhanced further. ${ }^{8}$ The result shows that the shape of NCs and the profile of their PDFs are correlated, even in the case of materials with highly symmetric internal atomic ordering. Therefore, PDFs can be used to assess the shape of NCs, for example, by comparing model PDFs for real-size NCs with those determined experimentally.

Third, various crystal phases including pure $\mathrm{Fe}_{3} \mathrm{O}_{4}$ (magnetite), $\alpha-\mathrm{Fe}_{2} \mathrm{O}_{3}$ (hematite), and $\gamma-\mathrm{Fe}_{2} \mathrm{O}_{3}$, as well as combinations of them, were tested and refined against the experimental PDF data. This was done by using structure models featuring continuous lattices of the respective structure types. The finite NC size was modeled by multiplying the model PDFs with a decaying exponent-type function, as implemented in the currently widely used software PDFFIT $^{11}$ for refinement of the internal atomic ordering of NCs. The finite-size models for NCs, shown in Figure 4, were not used to avoid mixing up of geometrical (size and shape) and atomic ordering type (e.g., unit cell constants, atomic positions) parameters. This approach guaranteed that the structure type parameters for polycrystalline and $\gamma-\mathrm{Fe}_{2} \mathrm{O}_{3} \mathrm{NCs}$ were kept the same and, therefore, their refined values could be compared in a straightforward manner. The PDF data for all three samples studied here were best approximated with a model based on the cubic (S.G. $F d \overline{3} m$ ) spineltype structure of $\gamma-\mathrm{Fe}_{2} \mathrm{O}_{3}$ (see Figure 3a) indicating that, within the limits of experimental accuracy, they all are single-phase NCs. The refined structure type parameters of polycrystalline, $\mathrm{SP}$ and TP $\gamma-\mathrm{Fe}_{2} \mathrm{O}_{3} \mathrm{NCs}$ are given in the SI. Their analysis shows that a larger than usual fraction of both octahedral and tetrahedral Fe-occupied sites in the NCs is vacant. As discussed in the literature $^{6 \mathrm{a}}$ this is due to the increased surface to volume ratio in NCs. This result could explain why the mass-normalized saturation magnetization of SPs and TPs is remarkably reduced when compared to that of polycrystalline $\gamma-\mathrm{Fe}_{2} \mathrm{O}_{3} .{ }^{4}$ The result again demonstrates the fact that atomic PDFs can be used to determine internal atomic ordering of NCs in the usual crystallographic terms of Bravais lattices, unit cells, atomic positions, and occupancies.

In summary, we have demonstrated that atomic PDFs can be used not only to determine the internal atomic ordering but also to assess the geometry of NCs. In particular, they provide very detailed structural information for $\gamma-\mathrm{Fe}_{2} \mathrm{O}_{3}$ spherical and tetrapod-shaped $\mathrm{NCs}$, which can be used to explain their growth mechanism ${ }^{4,10}$ as well as their magnetic properties. The approach can be very useful because it (i) employs a single experimental technique; (ii) is very flexible with respect to the NC state (e.g., solid, polymeric etc) and environment (e.g., low/high temperatures and pressures, solution, etc.) ${ }^{3}$ and (iii) can be performed either with laboratory equipment or at the state-of-the-art synchrotrons. ${ }^{3}$ Thus it has all the potential to become a standard "tool" for structural characterization in the rapidly developing field of nanoscience and technology.

Acknowledgment. Work at APS is supported by DOE under Contract DE-AC02-06CH11357. Benedetta Antonazzo is also thanked for help with the TP synthesis.

Supporting Information Available: Details on NC synthesis, TEM and XRD experiments, and structure modeling. This material is available free of charge via the Internet at http://pubs.acs.org.

\section{References}

(1) (a) Klug, H. P.; Alexander, E. In X-ray diffraction procedures for poly crystalline and amorphous materials; John Wiley \& Sons: 1974. (b) Rietveld, H. J. Appl. Crystallogr. 1969, 2, 65-71.

(2) (a) Murray, C. B.; Noris, D. J.; Bawendi, M. G. J. Am. Chem. Soc. 1993, 115, 8706-8715. (b) Hall, B. D. J. Appl. Phys. 2000, 87, 1666-1675.

(3) (a) Egami, T.; Billinge, S. J. L. In Underneath the Bragg peaks; Pergamon Press: 2003. (b) Petkov, V. Mater. Today 2008, 11, 28-38.

(4) Cozzoli, P. D.; Snoeck, E.; Garcia, M. A.; Giannini, C.; Guargliardi, A.; Cervellino, A.; Gozzo, F.; Hernando, A.; Achterhold, K.; Ciobanu, N.; Parak, F. G.; Cingolani, R.; Manna, L. Nano Lett. 2006, 6, 1966-1972.

(5) Cornell, R. M.; Schwertmann, U. In The Iron Oxides: Structures, Properties, Occurrences and Uses, 2nd ed.; Wiley-VCH: Weinheim, 2003.

(6) (a) Jorgensen, J.-E.; Mosegaard, L.; Thomsen, L. E.; Jensen, T. R.; Hanson, J. C. J. Solid State Chem. 2007, 180, 180-185. (b) Greaves, C. J. Solid State Chem. 1983, 49, 325-333.

(7) Ergun, S.; Schehl, R. R. Carbon 1973, 11, 127.

(8) (a) Gilbert, B. J. Appl. Crystallogr. 2008, 41, 554-562. (b) Kodama, K.; Iikubo, S.; Taguchi, T.; Shamoto, S. Acta Crystallogr. 2006, A62, 444453.

(9) Gateshki, M.; Petkov, V.; Pradhan, S.; Vogt, T. J. Appl. Crystallogr. 2005, $38,772-779$.

(10) The surface of NCs is passivated by a monolayer of surfactant molecules used to control their growth evolution in the liquid phase and ensure structural stability. In our case, a substantial fraction of the NC surface should be terminated with $\mathrm{O}$ atoms belonging to the carboxylate groups of oleic acid and/or the diol moiety of hexadecane-1, 2-diol. The surface of polycrystalline $\gamma-\mathrm{Fe}_{2} \mathrm{O}_{3}$ standard is also terminated with $\mathrm{O}$ atoms. Since the surface to volume ratio is very small compared to NCs, the apparent oxygen "surplus" in polycrystalline $\gamma-\mathrm{Fe}_{2} \mathrm{O}_{3}$ is negligible.

(11) Proffen, Th.; Billinge, S. J. L. J. Appl. Crystallogr. 1999, 32, 572-575.

JA9067589 